\title{
'Triple Clear': A Systematic and Up-to-Date Surgical Process for Giant Cell Tumor of Bone Graded Companacci II and III with or without Pathological Fracture and Joint Invasion
}

\section{Yushan Wang ( $\mathbf{3 8 4 5 3 4 2 3 4 @ q q . c o m ) ~}$}

Second Hospital of Shanxi Medical University https://orcid.org/0000-0003-4846-689X

Qiaoqiao Tian

Second Hospital of Shanxi Medical University

Haoze Li

Second Hospital of Shanxi Medical University

Jian Li

Second Hospital of Shanxi Medical University

\section{Peng Ren}

Shanxi Medical University

Yi Feng

Second Hospital of Shanxi Medical University

\section{Research Article}

Keywords: giant cell tumours of bone (GCTB), triple clear (TC), segmental resection (SR), Musculoskeletal Tumour Society (MSTS)

Posted Date: July 7th, 2021

DOl: https://doi.org/10.21203/rs.3.rs-644977/v1

License: (c) (i) This work is licensed under a Creative Commons Attribution 4.0 International License. Read Full License 


\section{Abstract}

Objectives: 1) To describe a systematic process for giant cell tumours of bone (GCTB). 2) To compare the clinical effects of 'triple clear' (TC) and segmental resection (SR), bone grafts and bone cement.

Method: Patients with primary GCTB graded Companacci II and III who were treated with either SR ( $\mathrm{n}=$ 39) or TC $(n=41)$ were included. The pain level was determined by the Visual Analogue Scale. Limb function was determined by the Musculoskeletal Tumour Society (MSTS) score.

Result: The operating time was $135.7 \pm 38.4 \mathrm{~min}$ in the TC group and $174.2 \pm 43.0 \mathrm{~min}$ in the SR group $(P<0.05)$. The recurrence rates were $7.3 \%$ and $10.0 \%$, respectively $(P=0.37)$. The MSTS scores at three months after surgery were $19.8 \pm 1.5$ in the TC group and $18.8 \pm 1.5$ in the SR group. The MSTS scores at two years were $26.3 \pm 0.7$ and $24.2 \pm 1.6$, respectively $(P \unrhd 0.05)$.

Conclusion: TC is recommended for GCTB graded Companacci II-III and GCTB accompanied by pathological fracture or joint invasion. Bone grafts may be more suitable than bone cement in the long term.

\section{Synopsis}

This article introduced a systematic and up-to-date surgical procedure ('Triple Clear') for patients with giant cell tumor of bone. By reviewing the data, clinical effects of segmental resection and 'Triple Clear'(TC) were compared. And the effects of two implant materials, allogeneic bone and bone cement, were also investigated. Finally, We concluded that TC should be the first choice for GCTB, and that bone grafts might provide more benefits than bone cement in the long term.

\section{Introduction}

Giant cell tumours of bone (GCTB) are common benign bone tumours. However, compared with general benign tumours, GCTB have a greater risk of malignant transformation and metastasis. The commonly used treatments for GCTB are curettage combined with adjuvant therapy and segmental resection (SR) with prosthesis reconstruction. Considering that most affected patients are between 20 and 40 years of age, curettage combined with adjuvant therapy is a more acceptable approach. Therefore, effective adjuvant therapy has become a focus of GCTB research. In the residual cavity after the removal of most tumour tissues, the clearing of the remaining tumour cells is an important step. A variety of physical and chemical inactivation methods have been reported.

In 1910, Bloodgood ${ }^{1}$ first applied phenol after curettage and obtained a good clinical effect. In 1973, Marcove ${ }^{2}$ first reported the result of cryotherapy by liquid nitrogen. Johnston ${ }^{3}$ first reported adjuvant therapy with hydrogen peroxide in 1987 . Jones ${ }^{4}$ reported in 2006 that $95 \%$ ethanol used in the residual cavity resulted in a recurrence rate of $20 \%$. Later, electrocauterization, argon beam coagulation and hypertonic saline were also applied in adjuvant therapy, and a good prognosis was obtained ${ }^{5-7}$. Even 
though many inactivation methods have been investigated, an efficient and safe method has not yet been determined. To reach this goal, researchers have compared the clinical effects of the different methods ${ }^{7-}$ 9 . All the researchers recommended that clinicians choose inactivation methods that are safer and associated with fewer complications. Therefore, according to the relevant literature (Table 1), we have summarized a few key principles. 1) Careful scraping of the wall of the residual cavity is essential after tumour curettage. 2) Regarding inactivation methods, while some methods do not have an obvious therapeutic effect, electrotome thermocoagulation (ET) and anhydrous ethanol (AE) infusion can achieve low recurrence rates without chemical burns or systemic toxicity. The high incidences of complications (such as osteoarthritis, fracture and infection) associated with hypothermia resulting from liquid nitrogen exposure were also avoided. ET and AE achieved ideal clinical effects. However, no researchers have attempted to combine these two methods for the treatment of GCTB. In this study, we propose the combination of $A E$ infusion and ET and present a systematic and efficient surgical procedure.

\section{Materials And Method}

\section{General patient characteristics and selection criteria}

From 2012 to 2018, we collected 71 patients with GCTB who were treated at our institute (Table 2). The lesions of 4 patients were located in the proximal humerus, while the other lesions were all located around the knee joint. Of the 71 patients, 30 underwent segmental resection with prosthesis reconstruction, while 41 underwent curettage combined with adjuvant therapy ('Triple clear' surgery). Preoperative pathological diagnosis was confirmed by needle puncture. X-ray, computed tomography (CT) and magnetic resonance imaging (MRI) were routinely performed before surgery to determine the tumour size and its invasion of the soft tissues. Pulmonary CT was performed to determine the presence of pulmonary metastasis. The degree of pain was recorded by the visual analogue scale (VAS) preoperatively (Table 1). The inclusion criteria were as follows: 1) patients with GCTB graded Companacci II or III; 2) patients with single-site lesions; 3) patients who received SR or TC; 4) patients with a follow-up time of more than $24 \mathrm{~m}$; 5) consistent results in the preoperative and postoperative pathological examinations; 6) no suspicious metastases; and 7) all lesions were primary. The exclusion criteria were as follows: 1 ) follow-up time less than $24 \mathrm{~m}$ or loss of follow-up information; 2 ) inconsistent results in the preoperative and postoperative pathological examinations; 3) suspicious metastases; and 4) recurrent lesions. The study conformed to the tenets of the Declaration of Helsinki 1964, and subsequent amendments and written informed consent were obtained from patients or their legal guardians.

\section{Surgical technique}

'Triple clear' surgery

'Triple clear' (TC) surgery is a systematic surgical procedure for GCTB that was developed through continuous exploration, clinical practice and relevant research worldwide. TC is applicable for GCTB 
graded Companacci I-III, GCTB with pathological fracture and damage to the joint surface. After anaesthesia, an incision was made to expose the lesion thoroughly. The soft tissues around the lesion were strictly protected. Considering the size of the lesion and postoperative mechanical strength, an appropriately sized bone window was made (Fig. 1A). The macroscopic tumour tissues were removed with a large curette, and the wall of the residual cavity was initially scraped. Then, middle- and smallsized curettes were successively used to repeatedly scrape the wall inch by inch (Fig. 1B). In this process, a dental endoscope was applied during the orthopaedic surgery to allow for clear observation of the cavity wall (Fig. 1C). Overall, there were no 'blank areas' observed during any of the operations. Highpressure flushing guns were then used to wash the residual cavity more than 3 times after scraping. It was ensured that the unknown tissues that had been scraped from the wall and the remaining tumour tissues were all removed. Subsequently, AE was used to infuse the residual cavity for 15 min (Fig. 1D). For the residual cavities with pathological fractures or invasion to the joint surface or surrounding soft tissues, we used AE to smear the wall and soft tissues that had been invaded. Then, the AE was aspirated. The residual cavity and surrounding tissues were rinsed again. Subsequently, with the assistance of the endoscope, the wall of the residual cavity and invaded soft tissues were cauterized inch by inch (Fig. 1E). The electrotome was used in electrocoagulation mode, and the power was $60 \mathrm{~W}$. The cauterization time at each point was 1-2s. For the wall close to the articular surface, we did not change the cauterization power, but reduced the cauterization time. After the burnout procedure, the residual cavity was rinsed for a third time (Fig. 1F) to flush out the carbonized tissues. Finally, the residual cavity was fully filled with bone cement or allografts (Fig. 1G). Finally, the bone window was closed and fixation was performed with plates and screws (Fig. 1H). After the negative-pressure drainage tube was placed, tissues were sutured layer by layer.

Segmental resection with prosthesis reconstruction

Before the application of "triple clear" surgery, segmental resection (SR) with prosthesis reconstruction was chosen for GCTB graded Companacci III and GCTB with pathological fracture or joint involvement to ensure a low recurrence rate. The procedure is briefly described below. Tissue separation was performed successively by appropriate approaches. After the relevant vessels and nerves were properly freed, osteotomy was performed $3 \mathrm{~cm}$ away from the edge of the lesion, and frozen margin examination was conducted. The combined artificial prosthesis was installed at the appropriate position, and functional tendons were connected to the prosthesis. Finally, the drainage tube was placed, and tissues were sutured layer by layer.

\section{Postoperative management and follow-up}

Prophylactic anti-infection treatment was administered for $72 \mathrm{~h}$. The degree of pain was recorded using VAS. When the local drainage volume was less than $50 \mathrm{~mL}$, the drainage tube was removed, and the patients were transferred to the rehabilitation department for rehabilitation. The rehabilitation process varied according to the surgery the patients had received. Patients who received TC only needed absolute immobilization for 3 days. After 3 days, they were able to carry out some mild muscular activities in bed. A series of passive progressive movements were started one week after surgery. Then, some active joint 
movements were added. Walking exercises with double crutches or weight training began when patients were able to raise the leg in a straight position. Patients were recommended to walk alone after 3 weeks and return to normal walking after 1 month. For patients who received SR with prosthesis reconstruction, the rehabilitation process was often painful and lengthy. For patients whose lesions were located at the proximal humerus, absolute immobilization of the upper limb was required for $1 \mathrm{w}$. For patients whose lesions were located at the distal femur, two-week absolute immobilization was recommended ${ }^{10}$. For patients whose lesions were located at the proximal tibia, the period of absolute immobilization was $4 \mathrm{w}$. After the absolute immobilization period, patients could carry out muscular activities and passive rehabilitation training. At 6 weeks postoperatively, patients were encouraged to undergo progressive weight training. Most patients returned to approximately normal levels at 3 months.

After patients were discharged from the hospital, routine follow-up procedures began, including the following: 1) radiography every 3 months to 2 years followed by biannual radiographs for 4 years and annual radiographs in the following years to evaluate oncological prognosis, 2) pulmonary CT biannually to monitor metastasis for 5 years and annually in the following years, 3 ) evaluation by the MSTS scoring system ${ }^{11}$ at 3 months and 2 years after surgery, and 4) evaluation of complications.

\section{Statistical analysis}

SPSS software version 20.0 (IBM Corp., Armonk, NY) was used for data analysis. Quantitative data are expressed as the mean \pm standard deviation, while qualitative data are expressed as the frequency. Independent sample t-test was used to compare the mean among

the two groups, and the chi square test or Fisher's exact test was used to compare the rates. $P<0.05$ was considered statistically significant.

\section{Result}

\section{Patient characteristics}

The mean age of the patients in the TC group was $33.1 \pm 12.6$. The mean age of the patients in the SR group was $38.6 \pm 15.4$. There was no significant difference in age or sex between the two groups. There were 21 patients graded Companacci II and 20 patients graded Companacci III in the TC group (Fig. 2), of whom 4 had pathological fractures. In the SR group, 9 patients were graded Companacci II, and 21 patients were graded Companacci III, of whom 7 had pathological fractures. There was also no significant difference in the condition of the patients between the two groups.

\section{Surgery and prognosis}

The mean operating time for TC was $135.7 \pm 38.4 \mathrm{~min}$ and that for SR was $174.2 \pm 43.0 \mathrm{~min}$. The operating time for TC was significantly shorter than that for SR $(P<0.05)$. The follow-up times were 54.7 \pm 23.2 months and $59.1 \pm 19.5$ months, respectively. There was no difference in the follow-up time 
between the two groups. Three patients in the TC group developed local recurrences during the follow-up, and all of them underwent a second operation. One patient underwent a second TC surgery and has not developed a second recurrence since then. The other two patients received SR. Among them, one patient did not develop recurrence, while the other patient developed multiple metastases in the lung six months after surgery and eventually died. Three patients in the SR group developed local recurrences, and all of them underwent amputations. After the second operation, two patients had no recurrence, while the other patient died shortly after surgery due to multiple metastases. The recurrence rate was $7.3 \%$ in the TC group and $10.0 \%$ in the SR group. There was no significant difference between the two groups. Of the eleven patients with pathological fractures, one patient in the SR group developed recurrence, while one patient in the TC group also developed recurrence. There was no significant difference in recurrence rates between the two groups in patients with pathological fracture. The postoperative VAS scores were significantly reduced in both groups, and there was no difference between the two groups. At 3 months postoperatively, the mean MSTS scores of the TC group and SR group were $19.8 \pm 1.5$ and $18.8 \pm 1.5$, respectively. The limb function of patients in the TC group was significantly better than that of those in the SR group at 3 months after surgery $(P<0.05)$. Moreover, the limb function of patients in the TC group was still better than that in the SR group at two years after surgery $(P<0.05)$. During follow-up, three patients in the SR group presented prosthesis loosening and underwent a second revision surgery. One patient in the TC group presented with a superficial infection in the incision. After sufficient drainage, sterilization and anti-infection treatment, the infection was effectively controlled. During the follow-up, joint stiffness, osteoarthritis, and other joint complications were not found. One patient in the TC group was dissatisfied with the procedure, while six patients in the SR group were dissatisfied. There was a significant difference in the degree of satisfaction between the two groups. $(P<0.05)$ (Table 3$)$.

\section{Filling materials}

The postoperative effects of the two filling materials, bone graft (BG) and bone cement (BC), were analyzed and compared. The mean MSTS score was $20.0 \pm 1.5$ in the BG group and 19.6 \pm 1.6 in the BC group at 3 months after surgery. At 2 years after surgery, the mean MSTS scores were $26.4 \pm 0.6$ and 26.4 \pm 0.7 , respectively. There was no significant difference between the two groups at either time point, which indicated that the two filling materials had no effects on the recovery of limb function. There was also no significant difference in recurrence rates between the two groups. In the BC group, seven patients presented with joint discomfort after surgery, which was statistically significant compared to number of patients with joint discomfort in the BG group. Related complications of the adjacent joint were not found during the follow-up (Table 4).

\section{Discussion}

Since Heijden ${ }^{12}$ and other scholars suggested treating GCTB with curettage, burring and adjuvant therapy in 2014 , the local recurrence rates have decreased from $30 \%-50 \%{ }^{12,13}$ to $6 \%-25 \%^{14,15}$. However, due to the use of numerous methods of inactivation, the clinical effects vary. Therefore, we proposed a systematic and efficient intraoperative management protocol for GCTB Companacci II and III based on the literature 
and our own experience. According to the results, the overall operating time for TC was significantly shorter than that for SR with prosthesis reconstruction, which resulted in a lower rate of intraoperative bleeding and a greater benefit to patients. A recurrence rate of $7.3 \%$ was achieved after TC, which is the lowest recurrence rate reported over the past decade ${ }^{7-9,16-20}$. However, Fraquet ${ }^{21}$ believed that segmental resection was the best choice for GCTB, including GCTB graded Companacci III, GCTB located at the distal radius, ulna, fibula and other non-load-bearing sites. The reason was that GCTB graded Companacci III is characterized by a large area of bone destruction and is bound to the articular surface, in some cases destroying the articular surface. However, after the improvement of the intraoperative process, we hypothesized that TC would also be suitable for GCTB graded Companacci III. According to the statistical results, we found that the recurrence rates after the two operations were similar, indicating that the effect of tumour cell elimination achieved by TC was similar to that achieved by SR. TC was even used in two patients whose lesions were located at the proximal humerus, and there was no recurrence observed in these two patients. Moreover, the patients maintained good upper limb function, which could not be achieved with SR. Some researchers believe that GCTB with pathological fracture is more likely to recur after surgery and, thus, SR should be chosen to reduce the recurrence rate. Our results indicated that even for patients with pathological fracture, the recurrence rates after SR and TC were similar. Moreover, TC could avoid the loss of autogenous articular tissue in young patients. Therefore, the acceptability of TC is obviously better than that of SR. In addition, patients in the TC group had more options for the second operation after recurrence, including a second curettage or SR. However, for patients in the SR group, amputation was likely the only remaining life-saving option. The choice of SR as the preferred treatment obviously deprives patients of the opportunity for limb preservation. However, for lesions with a wide range of joint damage, the joint surface is likely to collapse after the removal of tumour tissues, which will lead to leakage of articular fluid and a series of complications. Under these circumstances, TC is not applicable, and SR should be the preferred option.

In the procedure of TC, there were three key steps, as follows. 1) Scrape-out: Researchers generally agreed that a thorough curettage is the first and most important step. High-speed burring (HSB) has become a method highly praised by clinicians ${ }^{22}$. The required thickness of burring in different positions has been reported (approximately $1 \mathrm{~mm}$ into the normal cortical bone and $5 \mathrm{~mm}$ into the normal cancellous or subchondral bone $\left.{ }^{23}\right)$. However, in practice, it is difficult for to evaluate the thickness. Some researchers hypothesized that the sputtering of particles would cause the dissemination of tumour cells during $\mathrm{HSB}^{24}$. However, we do not think that the use of HSB will significantly reduce the recurrence rate in comparison with using curettes based on the fact that the scraping procedure used for TC is very careful and thorough. The tumour tissues could be easily removed by a large curette, and the wall of the residual cavity was scraped. The wall is not smooth; it contains many crests. After extensive burring with a large curette, medium and small curettes were used to subtly scrap the depressions. The whole process lasted at least $30 \mathrm{~min}$. A dental endoscope was used to check the wall and ensure that there was no residual tumour tissue. This is a very efficient innovation. All of the researchers agreed that the window that made to expose the lesion completely should be as large as possible. However, occasionally, the size of the lesion could not be accurately evaluated only by imaging and the extent of the residual cavity after 
scraping was much larger than that of the window. As a result, there were many blind areas that made it impossible to achieve complete scraping. Therefore, we chose to use a dental endoscope to solve this problem. The use of the endoscope allowed us to be sure that any operation we performed in the residual cavity was complete. 2.) Flushing out: A flushing gun was used to flush the residual cavity several times during the operation. The first wash was to wash off and remove the loose tissues on the surface of the scraped wall. The second wash was to dilute the remaining anhydrous ethanol in the residual cavity, which avoided the combustion of $A E$ during electrocauterization and further removed tumour tissues. The third irrigation was to remove the carbonized tissues and reduce the subsequent inflammatory response after electrocauterization. The frequency of each irrigation depended on the different conditions. 3.) Burn out: For adjuvant therapy, two of the safest methods (AE and electrocauterization) were used. We believe the effect of $A E$ infusion is superior to smearing $A E$. However, its applicability depends on whether infusion can be applied. Infusion the residual cavities with articular surface defects or pathological fractures is not recommended. Infusion is also not applicable to invaded soft tissues. The leakage of AE to the peripheral and intra-articular spaces is likely to cause injury to the vessels and nerves along with other complications. The Electromes in the electrocoagulation mode were used for cauterization at a power of $60 \mathrm{~W}$. The temperature was estimated to reach a range of $150{ }^{\circ} \mathrm{C}$ to $200{ }^{\circ} \mathrm{C}$. This temperature is far beyond the minimum temperature $\left(50^{\circ} \mathrm{C}^{25}\right)$ that can cause the necrosis of tissues. After $\mathrm{AE}$ infusion and cauterization, We believe that the combination of $\mathrm{AE}$ infusion and cauterization achieves deeper inactivation than $\mathrm{AE}$ or electrocauterization alone. Thus, with this method, the tumour tissues can be eliminated more thoroughly. The process was also applicable to invaded soft tissues. However, it is noted that for the wall close to the articular surface, the cauterization time should be reduced appropriately. Because the cortex of the articular surface is thin, extra-high temperatures and long heating times are likely to cause damage to chondrocytes. This damage is often irreversible, which will lead to serious joint complications.

For implant materials, we used to choose bone cement. The results of many previous studies indicated that the polymerization heat from bone cement could further inactivate tumour cells and further reduce the recurrence rate ${ }^{26}$. Moreover, bone cement also provides good mechanical strength after surgery. However, according to the statistical results, we found that there was no significant difference in the recurrence rate between the $B C$ group and the $B G$ group, which might be because the polymerization heat effect of $\mathrm{BC}$ after high-temperature inactivation by electrocoagulation was negligible. We also compared the functional recovery between the two groups and found that the functional recovery was not affected by the implant. In the BG group, no fracture was found during routine functional exercise after surgery, indicating that bone grafting could also fully support the patients' postoperative functional rehabilitation after strong internal fixation. However, in the BC group, we found that some patients had discomfort around the joint. Considering that bone cement may cause long-term complications such as osteoarthritis, allogeneic bone grafts have been increasingly chosen as the filling material. During the follow-up, we observed an obvious bone repair response in the residual cavity after bone grafts (Fig. 2F). This self-repair and spontaneous fusion will lead to greater comfort and better functional recovery in the 
long term. Later, with the exploration and application of biomaterials, bone grafts with osteoinductive biomaterials may be a better choice for clinicians.

For the follow-up of limb function, two time points ( 3 months and 2 years after surgery) were selected. Three months after surgery is a transitional time point for the recovery of limb function, while two years is a stable point. At the two time points, we found that the limb function of patients in the TC group was superior to that of patients in the SR group. In addition, the rehabilitation training for patients in the TC group was much easier, and the recovery period was shorter, which indicated that the retention of autologous joints had a beneficial influence on postoperative functional recovery. On the premise of the analogously low recurrence rates of the two surgical methods, the degree of satisfaction in patients in the TC group was higher than that in patients in the SR group. Since patients with GCTB are mostly young and middle aged, long-term complications caused by artificial joints can be expected. During the followup, 3 of 19 patients underwent revision surgeries. The high possibility of revision surgery after SR is another financial and emotional burden for patients. At present, except for superficial infection in one patient, we have not found any operation-related complications in the TC group, which may be due to the insufficient follow-up period. More patients and longer follow-up periods are needed to completely analyze the pros and cons of TC.

In both the SR and TC groups, one patient experienced relapse and died due to multiple metastases. At least two rigorous pathological examinations were performed on both patients, and the results were consistent. The rate of metastasis of GCTB is $1.5 \%$. The cellular and molecular biological aspects of this potential invasiveness are unknown. Therefore, we can only assess the state of tumour cells indirectly through patient characteristics. In the management of patients with GCTB, personal characteristics may also influence the choice of surgery. We emphasize the importance of repetitive pathological examinations. The aim of repetitive pathological examinations is not only to further confirm the diagnosis but also to detect changes in the course of the disease. The malignant transformation of GCTB is common. Once malignant transformation occurs, curettage within the lesion is absolutely not recommended, as it will lead to the artificial spread and metastasis of tumour cells. Disease progression caused by the wrong surgical choice can be catastrophic and even fatal; thus, follow-up is a critical aspect of patient management. If large recurrent lesions appear in a short period and progress rapidly, the possibility of malignant transformation should be considered first. Under these circumstances, SR should be chosen as the treatment option to avoid the worst outcome, even if the images still show the characteristics of benign tumours.

\section{Conclusion}

'Triple clear' surgery achieved a similar recurrence rate to segmental resection. Meanwhile, TC provided a shorter operation time, less blood loss, lower impact on functional recovery, and avoided secondary revision operations, which was of great benefit to patients. 
Therefore, 'triple clear' surgery is preferred for GCTB graded Companacci II-III and GCTB accompanied by pathological fracture or slight articular surface invasion. For the treatment of recurrent GCTB, the possibility of malignant transformation should also be considered as a factor in the choice of operations. In addition, allogeneic bone grafts may be a more suitable implant material than bone cement in the long term.

\section{Declarations}

\section{Ethics approval and consent to participate}

The study was approved by the ethics committee of the Second Hospital of Shanxi Medical University, and the requirement for patient consent was waived because of the retrospective nature of the study.

\section{Consent for publication}

All patients consented to the publication of the results of this study.

\section{Availability of Data and Materials}

The data used to support the findings of this study are available from the corresponding author upon reasonable request.

\section{Competing interests}

The authors declare that they have no competing interests.

\section{Funding}

No

\section{Authors' contributions}

Dr. Yushan Wang participated in all aspects of the study, including data collection, data screening and analysis, chart editing and manuscript writing. Dr. Qiaoqiao Tian and Jian Li collected the relevant data. Dr. Haoze Li processed the figures. Dr. Peng Ren conducted statistical analysis on the collected data and assisted in the preparation of the tables. My tutor, Professor Yi Feng, guided the research and put forward many key questions in it. 


\section{Acknowledgements}

Thanks to Dr. Jia Lv and Dr. Junjun Bai for their help during the intraoperative photo shooting. Thanks to Dr. Wangzhe Yang and Dr. Kun Zhang for their help in data and picture collection. The people above have given us permission to mention their names in the acknowledgments section.

\section{References}

1. Bloodgood JC. I. Benign bone cysts, ostitis fibrosa, giant-cell sarcoma and bone aneurism of the long pipe bones: a clinical and pathological study with the conclusion that conservative treatment is justifiable. Ann Surg. 1910;52(2):145-85.

2. Marcove RC, Lyden JP, Huvos AG, et al. Giant-cell tumors treated by cryosurgery. A report of twentyfive cases. J Bone Joint Surg Am. 1973;55(8):1633-44.

3. Johnston J. Treatment of giant-cell bone tumors by aggressive curettage and packing with bone cement. In Enneking WF, editor. New York: Limb Salvage in Musculoskeletal Oncology. Churchill Livingstone. 1987: 512-516.

4. Jones KB, DeYoung BR, Morcuende JA, et al. Ethanol as a local adjuvant for giant cell tumor of bone. lowa Orthop J. 2006;26:69-76.

5. Ofluoglu O. Aggressive treatment of giant cell tumour with multiple local adjuvants. Acta Orthop Belg. 2008;74(6):831-6.

6. Lewis VO, Wei A, Mendoza T, et al. Argon beam coagulation as an adjuvant for local control of giant cell tumor. Clin Orthop Relat Res. 2007;454:192-7.

7. Benevenia J, Patterson FR, Beebe KS, et al. Comparison of phenol and argon beam coagulation as adjuvant therapies in the treatment of stage 2 and 3 benign-aggressive bone tumors. Orthopedics. 2012;35(3):e371-8.

8. Lin WH, Lan TY, Chen CY, et al. Similar local control between phenol- and ethanol-treated giant cell tumors of bone. Clin Orthop Relat Res. 2011;469:3200-8.

9. Lizz van der Heijden, Ingrid CM, van der Geest, et al. Liquid Nitrogen or Phenolization for Giant Cell Tumor of Bone? A Comparative Cohort Study of Various Standard Treatments at Two Tertiary Referral Centers. J Bone Joint Surg Am. 2014;96:35(1-9).

10. Malawer MM, Sugarbaker PH. Musculoskeletal cancer surgery: treatment of sarcomas and allied diseases. Springer Science \&Business Media; 2001.

11. Enneking WF, Dunham W, Gebhardt MC, Malawar M, Pritchard DJ. A system for the functional evaluation of reconstructive procedures after surgical treatment of tumors of the musculoskeletal system. Clin Orthop Relat Res. 1993; 241-246.

12. Trieb K, Bitzan P, Dominkus M, et al. Giant-cell tumors of long bone. J Bone Joint Surg Am. 2000;82(9):1360-1. 
13. Karpik M. Giant cell tumor (tumor gigantocellularis, osteoclastoma)-epidemiology, diagnosis, treatment. Ortop Traumatol Rehabil. 2010;12(3):207-15.

14. Szendröi M. Giant-cell tumour of bone. J Bone Joint Surg Br. 2004;86(1):5-12.

15. Turcotte RE. Giant cell tumor of bone. Orthop Clin North Am. 2006;37(1):35-51.

16. Errani C, Ruggieri P, Asenzio MA, et al. Giant cell tumor of the extremity: A review of 349 cases from a single institution. Cancer Treat Rev. 2010;36:1-7.

17. Klenke FM, Wenger DE, Inwards CY, et al. Giant cell tumor of bone: Risk factors for recurrence. Clin Orthop Relat Res. 2011;469:591-9.

18. Akihiko Takeuchi P, Suwanpramote N, Yamamoto T, Shirai. Katsuhiro HayashiHiroaki Kimura. Mid- to long-term clinical outcome of giant cell tumor of bone treated with calcium phosphate cement following thorough curettage and phenolization. J Surg Oncol. 2018;1-7.

19. Jamshidi Khodamorad Z, Farshad, Haji Agha Bozorgi Milad et al. Extended curettage versus en bloc resection for the treatment of grade 3 giant cell tumour of the knee with pathologic fracture: a retrospective study.[J].Int Orthop, 2021, 45: 289-297.

20. Liu Qing,He Hongbo,Yuan Yuhao. et al. Oncology and functional prognosis are both vital in the surgical treatment of RGCTs around the knee joint.[. J]Am J Transl Res. 2020;12:1155-65.

21. Fraquet N, Faizon G, Rosset $P$, et al. Long bones giant cells tumors: treatment by curretage and cavity filling cementation. [J] Orthop Traumatol Surg Res. 2009;95:402-6.

22. Machak Gennady N, Snetkov Andrey I. The impact of curettage technique on local control in giant cell tumour of bone.[. J]Int Orthop. 2021;45:779-89.

23. Jamshidi K, Shirazi MR, Bagherifard A, Mirzaei A. Curettage, phenolization, and cementation in paediatric Ewing's sarcoma with a complete radiological response to neoadjuvant chemotherapy. Int Orthop. 2019;43(2):467-73.

24. Wang Pai-Han,WuChia-Lun,Chen Chao-Ming, et al. Adjuvant therapy by high-speed burr may cause intraoperative bone tumor seeding: an animal study.[J].BMC Musculoskelet Disord, 2020, 21: 507.

25. Nelson CG, Krishnan EC, Neff JR. Consideration of physical parameters to predict thermal necrosis in acrylic cement implants at the site of giant cell tumors of bone. Med Phys. 1986;13(4):462-8.

26. Gupta Som P. Garg Gaurav,Curettage with cement augmentation of large bone defects in giant cell tumors with pathological fractures in lower-extremity long bones.[. J]J Orthop Traumatol. 2016;17:239-47.

\section{Tables}

Due to technical limitations, table 1 to 4 is only available as a download in the Supplemental Files section.

\section{Figures}



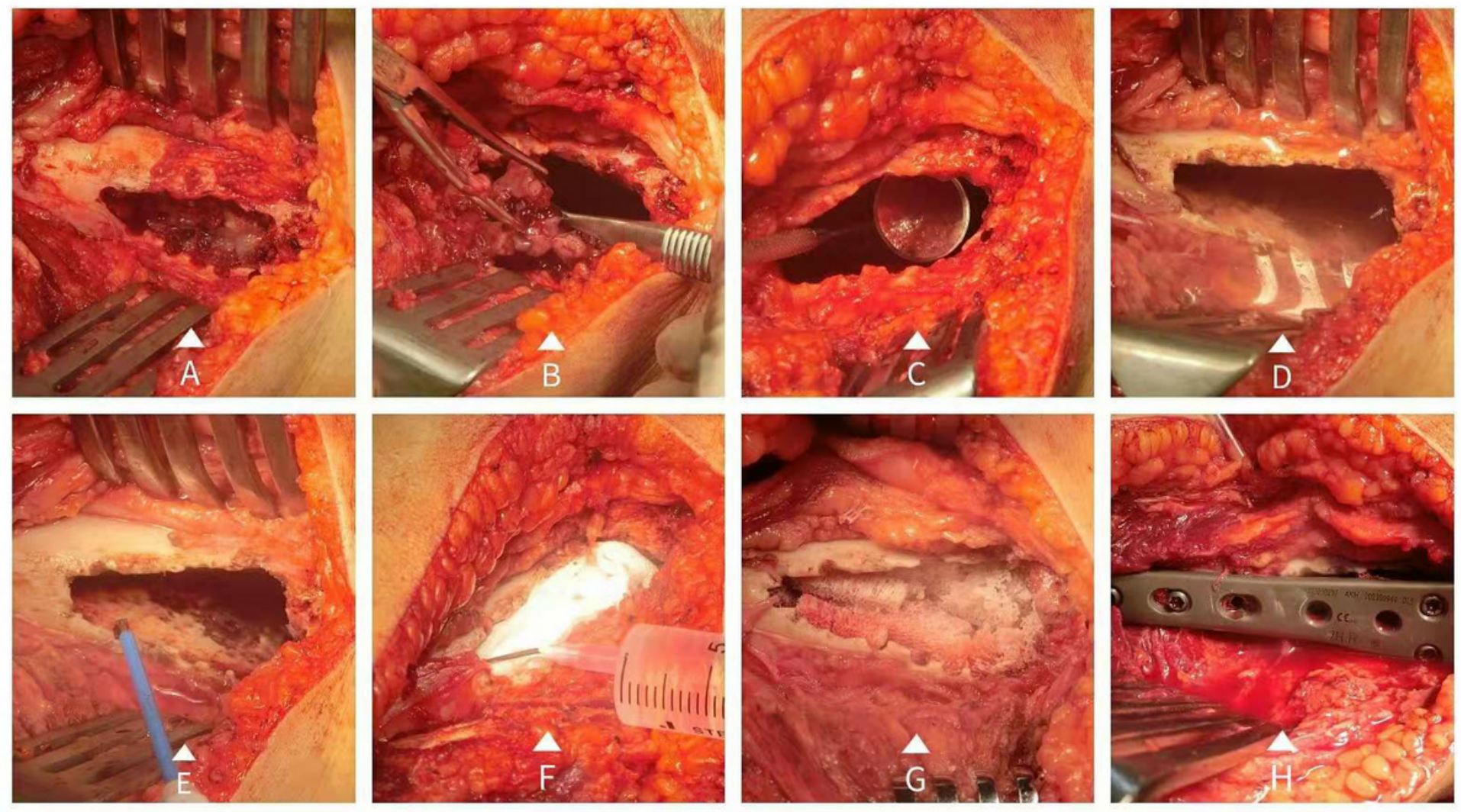

\section{Figure 1}

The procedure of TC. A: A bone window of appropriate size was opened in the area of the lesion. B: The tumor body was scraped and the residual cavity wall was polished with different types of spatulas in order. C: Dental endoscopy was used to examine the residual cavity and clear the visual dead corners. D: The residual cavity was soaked with anhydrous ethanol for $15 \mathrm{~min}$. E: Use an electric knife to cauterize the wall of the residual cavity inch by inch. F: In the final rinse, we used gauze to fill the residual cavity and then injected water, so as to better take out the carbonized necrotic tissues on the wall. G: Allogeneic bones are compactly inserted into the cavity. $\mathrm{H}$ : The bone window was reset with plates and screws. 

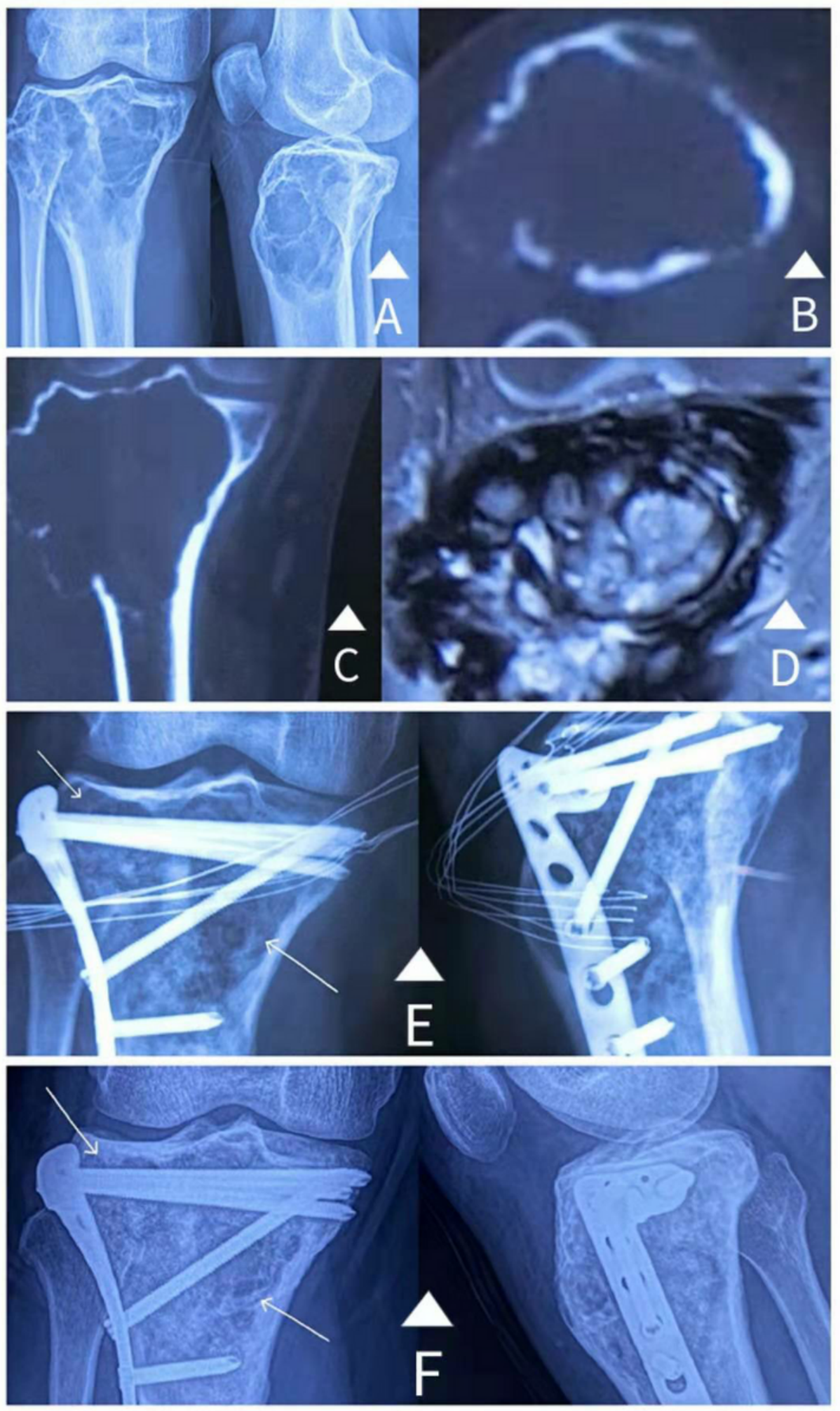

\section{Figure 2}

A: Preoperative anteroposterior and lateral radiographs of the proximal tibia showed local bone destruction, expansive growth, and perforation changes. B: The transection film of CT showed that the tumor had broken through the bone cortex and invaded the surrounding soft tissue. The boundaries are not clear. It is graded as Companacci III. C: Coronal CT images were used to further evaluate the tumor size. D: MRI showed mixed signals in the tumor. E: Radiographs on the first day after surgery showed a 
sparse local bone mass with many 'blank areas'(arrow). F: Radiographs one year after surgery showed that local bone mass significantly increased. The previous 'blank areas' presented significant bone repair response. The transplanted allografts fused well with the new bone tissues.

\section{Supplementary Files}

This is a list of supplementary files associated with this preprint. Click to download.

- Table1.pdf

- Table2.pdf

- Table3.pdf

- Table4.pdf 